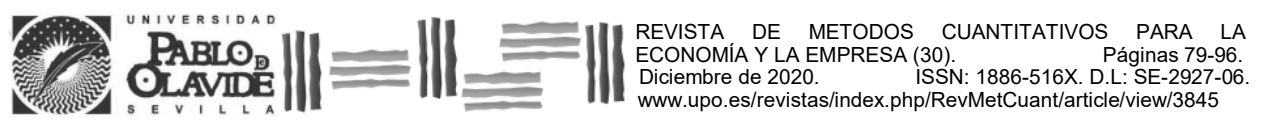

\title{
Toma de Decisiones Estratégicas en Entornos Inciertos
}

\author{
BLANCO-MESA, FABIO \\ Facultad de Ciencias Económicas y Administrativas, Escuela de Administración de Empresas \\ Universidad Pedagógica y Tecnológica de Colombia, Tunja (Colombia) \\ Correo electrónico: fabio.blanco01@uptc.edu.co \\ LEÓN-CASTRO, ERNESTO \\ Facultad de Ciencias Económicas y Administrativas \\ Universidad Católica de la Santísima Concepción, Concepción (Chile) \\ Correo electrónico: eleon@ucsc.cl \\ Acosta-Sandoval, Alejandra \\ Facultad Sede Duitama \\ Universidad Pedagógica y Tecnológica de Colombia, Duitama (Colombia) \\ Correo electrónico: maria.acosta04@uptc.edu.co
}

\begin{abstract}
RESUMEN
El proceso de toma de decisiones tiene una incidencia relevante en los resultados de las empresas, lo que ha llevado a desarrollar novedosos métodos que permitan evaluar bajo condiciones no controlables elementos subjetivos y racionales. En ese sentido, el objetivo principal de este trabajo estudia los operadores de agregación en la toma de decisiones en entornos inciertos. Se presentan dos metodologías que permiten agregar información, que se llaman operadores OWA y BON-OWA. La aplicación de estos operadores se realiza en la selección de lanzamiento de nuevos productos. La principal ventaja de estos operadores es que permiten capturar la actitud del decisor y la comparación e interrelación continua de la información. Así, se destaca el análisis holístico que ofrecen estos métodos sobre la toma de decisiones en incertidumbre, que permite integrar conceptos de la teoría administrativa y la teoría de la agregación en un caso aplicado, visualizando como la inclusión de la información genera cambios dentro de los rankings de selección de alternativas.
\end{abstract}

Palabras clave: toma de decisiones, incertidumbre, operadores de agregación.

Clasificación JEL: C02; C43; D81; L1.

MSC2010: 18B35; 47A54.

Artículo recibido el 1 de febrero de 2019 y aceptado el 25 de junio de 2019. 


\title{
Strategic Decision-Making in Uncertain Environments
}

\begin{abstract}
Decision-making has a relevant incidence on firms results, which has led to develop novel methods that allow assessing subjective and rational elements under uncontrollable conditions. In this sense, the main aim of this work is to study aggregation operators in decision-making in uncertain environments. Two methodologies allow aggregating information are presented, which are called OWA and BON-OWA operators. The application of these operators is made in the selection of new product release. The main advantage of these operators is that they allow to capture the attitude of the decision maker and the continuous comparison and interrelation of the information. Thus, the holistic analysis offered by these methods on decision making in uncertainty is highlighted, which allows to integrate concepts of administrative and aggregation theories in an applied case, visualizing how the inclusion of information generates changes within the selection of alternatives.
\end{abstract}

Keywords: decision-making, uncertainty, aggregation operators.

JEL classification: C02; C43; D81; L1.

MSC2010: 18B35; 47A54.

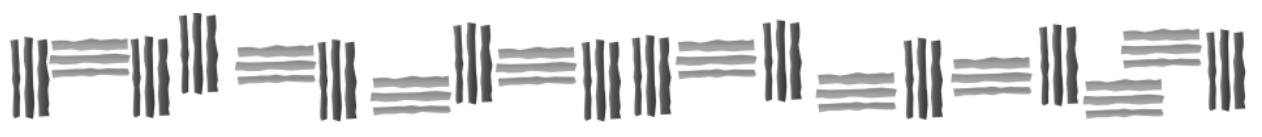




\section{Introducción.}

La toma de decisiones en entornos inciertos es un campo que ofrece resultados válidos a partir de la subjetividad y el razonamiento inspirado desde la lógica difusa (Zadeh, 1965). La lógica difusa analiza el concepto de los conjuntos borrosos, que permite usar los conjuntos Booleanos clásicos en una lógica de valores múltiples. Las investigaciones en la toma de decisiones en incertidumbre tienen origen en los estudios de los conceptos de conjuntos difusos (Zadeh, 1965), entornos difusos (Bellman \& Zadeh, 1970), razonamiento aproximado (Zadeh, 1975a; 1975b; 1975c) y aplicaciones de los conjuntos difusos en los sistemas de decisión (Zimmermann, 1986). Su principal argumento se basa en que un gran número de las decisiones en el mundo real ocurren en un entorno en el que las consecuencias de posibles acciones no son conocidas con precisión. En ese sentido, una decisión puede ser vista como una intersección de los objetivos y restricciones dadas dentro de un proceso de múltiples etapas donde la inteligencia humana tiene la habilidad de manipular conceptos difusos y responder instrucciones difusas (Bellman \& Zadeh, 1970). Dentro de las problemáticas que se han trabajado a través de metodologías difusas se encuentran decisiones financieras (Zeng \& Su, 2015), pronóstico del tipo de cambio (LeónCastro et al., 2018b), métodos alternativos de solución de conflictos (Pérez-Arellano et al., 2019), medición de la innovación (Alfaro-García et al., 2018), selección de productos (Merigó \& Gil-Lafuente, 2010), evaluación de proyectos (Doskéocil, 2015), análisis de clientes (Ortigosa-Hernández \& GilLafuente, 2016), entre muchas otras.

Una de las aproximaciones más estudiadas en este campo es el operador Ordered Weighted Average (OWA) como método utilizado para la toma de decisiones. Este método permite agregar la información y obtener un orden, en el que se muestran de forma descendente o ascendente las opciones evaluadas. A partir de este método se han creado múltiples extensiones que permiten ordenar, relacionar y diferenciar la información, uno de los cuales es el operador BON-OWA. Este operador combina las características del operador OWA (Yager, 1988) y la media de Bonferroni (Bonferroni, 1950). La media de Bonferroni permite establecer la relación de dos medias aritméticas y el producto. Así, el BONOWA permite interrelacionar, comparar continuadamente y ordenar la información.

En ese sentido, el presente trabajo tiene como objetivo principal estudiar los operadores de agregación en la toma de decisiones en entornos inciertos. Para alcanzarlo, se hará una revisión de la teoría de la decisión en entornos inciertos y se presentarán los conceptos básicos de los operadores OWA y BON-OWA como herramientas de agregación de información. Posteriormente, se presenta la aplicación de los operadores a través de un caso de selección de producto a lanzar al mercado de una empresa mexicana. En dicho caso, se evalúan cuatro diferentes productos que tienen la posibilidad de ser lanzados al mercado a través de seis elementos de evaluación seleccionados por la alta dirección de la organización y cada uno es evaluado por el área de desarrollo y operaciones de la empresa. Esta información es analizada a través de cuatro operadores distintos de agregación de información y se detalla cada uno de estos resultados. Finalmente, este estudio permite de manera holística observar una metodología novedosa sobre toma de decisiones en situaciones de incertidumbre, que integra conceptos de la teoría administrativa y la teoría de la agregación en un caso aplicado donde se visualiza cómo al agregar información las ordenaciones de las alternativas varían.

La actual investigación tiene la siguiente estructura. En la sección 2 se profundiza sobre la teoría de la toma de decisiones en incertidumbre, operadores OWA y BON-OWA y un caso práctico de los operadores. En la sección 3 se presenta un caso práctico de los operadores sobre selección de productos y la sección 5 resume las principales conclusiones del trabajo. 


\section{Teoría y métodos para la toma de decisiones en entornos inciertos.}

\subsection{Teoría de la toma de decisiones en incertidumbre.}

Ante un mundo globalizado caracterizado por la alta cantidad de información, cambios constantes e incertidumbre, los fenómenos relacionados con la realidad y la complejidad humana son cada vez menos previsibles y más inciertos, difusos y difíciles de captar (Gil-Aluja, 1999; Yager, 1996b; 2006). Esto ha llevado al desarrollo de estudios que pueden combinar metodologías que traten la incertidumbre y la probabilidad con el fin de obtener secuencias y escenarios significativos de los hechos que ocurren en la realidad.

Es así como inspirados en la lógica difusa (Zadeh, 1965), se han utilizado de forma relevante dentro del análisis de toma de decisiones, con la finalidad de que éstas se acerquen cada vez más a las realidades que viven las personas/organizaciones/países/mundo. Dentro de los trabajos que se han realizado al respecto se encuentran los realizados con conjuntos difusos (Klir \& Yuan, 1995; Garg, 2016), conjuntos vagos (Chen \& Tan, 1994; Hong \& Choi, 2000), conjuntos difusos intuicionistas (Lin et al., 2007), técnica para el orden de preferencias por similitud con la solución ideal (TOPSIS) (Chen, 2000; Boran et al., 2009), entre otras.

Su principal argumentación se basa en que un gran número de las decisiones en el mundo real ocurren en un entorno, en el que las consecuencias de posibles acciones no son conocidas con precisión. En ese sentido, una decisión puede ser vista como una intersección de objetivos y restricciones dadas dentro de un proceso de múltiples etapas donde la inteligencia humana tiene la habilidad de manipular conceptos difusos y responder instrucciones difusas (Bellman \& Zadeh, 1970).

Basado en lo anterior, en la toma de decisiones se ha pasado del concepto de probabilidad al de posibilidad destacando diferencias importantes entre ellas. Según Blanco-Mesa (2015) son dos términos que se utilizan indistintamente para interpretar la realidad al etiquetar eventos como probables, cuando debería decirse qué realidad es posible. Por un lado, la probabilidad está relacionada con el azar, la aleatoriedad, la medida y lo objetivo soportado por un conjunto de leyes para explicar la realidad con hechos del pasado. Por otro lado, la posibilidad está relacionada con lo difuso, las valuaciones, lo subjetivo, la percepción y la sensación, donde la realización de los hechos no se puede ubicar en el tiempo y en el espacio, el pasado no aporta información suficiente y las acciones presentes son valuadas para la previsión de los acontecimientos.

Bajo esta lógica se ha creado un marco teórico para el análisis de la información de forma posibilista y análoga, en donde la información debe incorporarse dentro de la medición (Zadeh, 1999). Esta actitud hacia el análisis de la información de forma análoga y la incertidumbre del comportamiento humano es la que ha llevado al estudio de un nuevo campo de análisis de decisión (Chen \& Hwang, 1992). En ese sentido, el desarrollo de este campo de investigación se centra en el tratamiento de problemas de toma de decisiones de múltiple criterio (MCDM, siglas en inglés para Multiple Criteria Decision Making), en los que se tiene en cuenta la subjetividad del decisor para seleccionar, priorizar, y ordenar diferentes acciones y observar la factibilidad de una alternativa de acuerdo a los recursos disponibles (Blanco-Mesa et al., 2017).

Así, las metodologías de la lógica difusa son combinadas con la MCDM para el tratamiento de los problemas en situaciones de incertidumbre y alta subjetividad, ya que pueden involucrar variables lingüísticas y variables difusas al momento de definir metas, pros y contras de las características de las decisiones (Tzeng \& Huang, 2011). Un aspecto a destacar es que los problemas de MCDM pueden ser clasificados en dos: a) toma de decisiones con atributos múltiples (MADM, siglas en inglés para Multiple Attribute Decision Making) y b) toma de decisiones con múltiple objetivo (MODM, siglas en inglés para Multiple Objective Decision Making) (Hwang \& Yoon, 1981). Dentro de las MADM, el número de alternativas ya han sido establecidas y el proceso de toma de decisiones selecciona, prioriza y ordenar un número finito de acciones de curso. En las MODM el principal interés se enfoca en diseñar la alternativa "más" posible en relación a la limitación existente de los recursos disponibles (Chen \& 
Hwang, 1992). Recientemente, Xu (2015) ha propuesto una nueva categoría llamada la toma de decisiones inciertas con atributos múltiples (UMADM, siglas en inglés para Uncertain Multiple Atributte Decision Making), que permite ordenar y priorizar la información de acuerdo a su peso. Las UMADM usan operadores de agregación como la media ponderada (WA) (Harsanyi, 1955), la media ponderada ordenada (OWA, siglas en inglés para Ordered Weighted Average) (Yager, 1988) y la media ponderada híbrida (HWA) (Xu, 2008).

Así, con el desarrollo de los diferentes métodos para la toma de decisiones son muchas las aplicaciones y aproximaciones que se han propuesto en áreas como las ciencias computacionales, ingeniería, ciencia de la gestión de operaciones, matemáticas, negocios económicos y sistemas de control automático. Asimismo, estos nuevos métodos son aplicados a problemas empresariales actuales como la gestión de la cadena de suministros, toma de decisiones en inversiones, evaluación de personal, rediseño de productos y servicios de mantenimiento (Blanco-Mesa et al. 2017).

\subsection{El operador OWA como método para la toma de decisiones.}

Actualmente, existen múltiples propuestas desarrolladas con la matemática de la lógica difusa para la toma de decisiones. En este trabajo profundizaremos en el operador OWA. Este método permite agregar la información y obtener ordenaciones de forma descendente o ascendente de las opciones evaluadas. Las investigaciones desarrolladas bajo este método abarcan campos como la ingeniería, informática, economía y estudios en empresa, en los que se observan avances teóricos, matemáticos y aplicaciones prácticas. Las aplicaciones teóricas van dirigidas al desarrollo de nuevos algoritmos y a la integración de métodos para la ordenación de la información. Dentro de las aplicaciones y algoritmos desarrollados se destacan los hechos en el campo de los estudios empresariales, que abordan temas relacionados con las finanzas (Gil-Aluja, 1996; Merigó \& Gil-Lafuente, 2010; 2007; 2006), estrategia (Wei \& Merigó, 2012; Merigó \& Gil-Lafuente 2008; 2012; Merigó \& Casanovas, 2010; Vigier et al., 2017; Merigó, 2015), emprendimiento (Blanco-Mesa et al., 2015; 2018a), grupos de interés (Llopis \& PalaciosMarqués, 2017; Blanco-Mesa et al., 2018b; 2018c), gestión de los recursos humanos (Merigó \& GilLafuente, 2011; Canós et al., 2014; Canós \& Liern, 2008), gestión del riesgo empresarial (Blanco-Mesa et al., 2019) y mercadeo (Brijs et al., 2006). Estos estudios hacen importantes aportaciones metodológicas para el tratamiento de información proveniente de tomadores de decisiones siendo capaz de parametrizar su actitud y sus preferencias.

Dentro de los campos para la aplicación y el desarrollo de estos métodos en estudios empresariales es la toma de decisiones a nivel estratégico. En este proceso de toma de decisiones se involucra la intuición, la racionalidad y el conocimiento. La intuición involucra tareas y procesos complejos en cortos horizontes de tiempo con una alta carga de subjetividad y juicios de valor. La racionalidad es un proceso analítico, sistémico, basado en reglas y con mecanismos explícitos, que tienen una gran aceptación entre los tomadores de decisiones ya que siguen un proceso de toma de decisiones paso a paso. El conocimiento puede ser obtenido a través de la experiencia, la práctica, la capacitación y educación continuada, que a su vez es acumulado, compartido y replicado y puede ser usado en cualquier momento para dar soluciones a problemas. Por lo tanto, resulta pertinente profundizar en los estudios de toma de decisiones estratégicas en incertidumbre usando estos novedosos métodos, ya que permiten capturar la actitud del tomador de decisiones cuando la información disponible es insuficiente o confusa.

\subsection{Conceptos básicos del operador OWA.}

El operador OWA fue propuesto por Ronald Yager (1988). La finalidad de este operador es obtener un valor representativo a partir de la agregación de una serie de datos que reflejan los parámetros de optimismo/pesimismo predeterminados. Así, la información provista por cada tomador de decisiones evidencia su grado de optimismo o pesimismo. La definición del operador OWA es la siguiente. 
Definición 1: Un operador OWA se define como una función de dimensión $n$ es una aplicación $F: R^{n} \rightarrow R$, que está asociada en un vector de ponderación $W$ de dimensión $n W=\left[w_{1}, w_{2}, \ldots, w_{n}\right]$ que cumple con las siguientes condiciones:

- $\quad w_{j} \in[0,1]$

- $\quad \sum_{j=1}^{\ddot{2}} w_{j}=1$

- $\quad f\left(a_{1}, a_{2}, \ldots, a_{n}\right)=\sum_{j=1}^{n} w_{j} b_{j}$

La característica fundamental del operador OWA es el reordenamiento de los argumentos a través de la asociación con una ponderación, de acuerdo a $b_{j}$ que es el valor más alto (o bajo) de los argumentos $a_{1}, a_{2}, \ldots, a_{n}$. Así, la ordenación introduce la no linealidad en el proceso de agregación. A continuación, se mostrará un ejemplo matemático del procedimiento:

$$
W=[0.5,0.4,0.1,0.7]
$$

entonces:

$f(0.4,0.2,0.5,0.3)=(0.5) \times(0.5)+(0.3) \times(0.4)+(0.4) \times(0.1)+(0.2) \times(0.7)=0.55$

El operador OWA es una media ponderada que cumple con propiedades que definen a este método como un operador de medidas, cuyas características son las siguientes (Yager, 1988; 1996a; 2002):

a) Conmutativa: $f\left(a_{1}, a_{2}, \ldots, a_{n}\right)=f\left(d_{1}, d_{2}, \ldots, d_{n}\right)$;

b) Monotonía: $f\left(c_{1}, c_{2}, \ldots, c_{n}\right) \leq f\left(a_{1}, a_{2}, \ldots, a_{n}\right)$;

c) Delimitado: $\min \left\{a_{i}\right\} \leq f\left(a_{1}, \ldots, a_{n}\right) \leq \max \left\{a_{i}\right\}$;

d) Idempotencia: $f\left(a_{1}, a_{2}, \ldots, a_{n}\right)=a$;

e) Carácter actitudinal: $\alpha(W)=\sum_{j=1}^{n} w_{j}\left(\frac{n-j}{n-1}\right)$;

f) Entropía: $H(W)=-\sum_{j=1}^{n} \ln \left(w_{j}\right)$;

g) Operador de balance: $\operatorname{Bal}(W)=\sum_{j=1}^{n} \frac{(n+1-2 j)}{n-1} w_{j}$;

h) Grado de divergencia: $\operatorname{Div}(W)=\sum_{j=1}^{n} w_{j}\left(\frac{n-j}{n-1}-\alpha(W)\right)^{2}$.

Tradicionalmente, en la teoría de la decisión han existido varios criterios que permiten hacer una elección de alternativa en situaciones de incertidumbre. Una característica destacada de estos criterios es que se desconoce el resultado final, por lo que la actitud del tomador de decisiones juega un rol 
importante. A continuación se presentan algunos de estos criterios explicados por Casanovas et al. (2014):

a) Optimista: $w_{1}=1$ y $w_{j}=0, \forall_{j} \neq 1$, i.e. $F\left(a_{1}, \ldots, a_{n}\right)=\operatorname{Max}\left\{a_{j}\right\}$;

b) Pesimista: $w_{n}=1$ y $w_{j}=0, \forall_{j} \neq n$, i.e. $f\left(a_{1}, \ldots, a_{n}\right)=\operatorname{Min}\left\{a_{j}\right\}$;

c) Laplace: $w_{j}=\frac{1}{n}, \forall_{j} \neq 1$ i.e. $f\left(a_{1}, \ldots, a_{n}\right)=\frac{1}{n} \sum_{j=1}^{n} a_{j}$;

d) Hurwicz: $w_{1}=\alpha \quad w_{n}=1-\alpha$ y $w_{j}=0, \forall_{j} \neq 1$, i.e. $f\left(a_{1}, \ldots, a_{n}\right)=\alpha \operatorname{Max}\left\{a_{j}\right\}+(1-$ $\alpha) \operatorname{Min}\left\{a_{j}\right\}$.

Dada la flexibilidad que proporciona este algoritmo para la obtención de vectores de peso se han propuestos diversas familias, tales como maximal entropy (Me-OWA) (O'Hagan, 1990), Step-OWA (Yager, 1993), Window-OWA (Yager, 1993), S-OWA (Yager, 1994), Olympic-OWA (Yager, 1996a) y E-Z-OWA (Yager, 2003) que han permitido desarrollar múltiples extensiones de este operador en combinación con otros algoritmos que permiten comparar e interrelacionar la información.

\subsection{El operador Bonferroni-OWA.}

En el campo de la teoría de la agregación se han desarrollo una gran cantidad de métodos para la toma de decisiones en incertidumbre (Blanco-Mesa et al., 2017). En este trabajo se estudiará el operador Bonferroni-OWA y algunas de sus extensiones para su aplicación en la toma de decisiones en incertidumbre.

La media de Bonferroni involucra el producto de cada uno de los argumentos con la media del resto de los argumentos, es decir, es la relación de dos medias aritméticas y el producto. Éste es un método simple que permite que se realicen una gran cantidad de argumentos o que se construyan intervalos de confianza mientras se asegura que se mantenga un coeficiente de confianza global (Bonferroni, 1950). Así, este método permite hacer múltiples comparaciones entre cada argumento de entrada y captura su interrelación (Blanco-Mesa et al., 2016). Este operador se define de la siguiente manera:

$$
B\left(a_{1}, a_{2}, \ldots, a_{n}\right)=\left(\frac{1}{n} \frac{1}{1-n} \sum_{\substack{j=1 \\ j \neq k}}^{n} a_{j}^{q}\right)^{\frac{1}{r+q}}
$$

Una regla fundamental para poder aplicar la media de Bonferroni es que los argumentos $a$ deben ser mayores o iguales a 0 y que los parámetros $r, q$ deben ser mayores o iguales a 0 .

Yager (2009) propone un nuevo algoritmo que combina el operador OWA y la media de Bonferroni y sugiere una generalización de este operador. Este operador se define de la siguiente manera:

$$
\mathrm{BON}-\mathrm{OWA}\left(\mathrm{a}_{1}, \ldots, \mathrm{a}_{\mathrm{n}}\right)=\left(\frac{1}{n} \sum_{i} a_{i}^{r} O W A_{W}\left(V^{i}\right)\right)^{\frac{1}{\mathrm{r}+\mathrm{q}}}
$$


La característica fundamental de este operador combina el de los operadores presentados anteriormente. Adicionalmente, el vector $\left(V^{i}\right)$ contiene todos $\operatorname{los}$ argumentos $\mathrm{a}_{\mathrm{j}}$ excepto los $\operatorname{argumentos} \mathrm{a}_{\mathrm{i}}$.

\subsection{Caso práctico de los operadores OWA y BON-OWA.}

Para entender el funcionamiento de estos operadores y compararlos para observar sus resultados se presenta un ejemplo matemático ilustrativo. Este ejemplo sigue una secuencia de 5 pasos que permiten llevar el proceso de toma de decisión. Los pasos son los siguientes:

Paso 1: En este paso se determinan las alternativas y las características con las que se crearán los argumentos para ser ponderados.

Paso 2: Con las alternativas y las características determinadas se crea la matriz de argumentos $a_{i}$ para ser ponderados.

Paso 3: En el paso tres se determina el vector de peso $W$ para cada uno de los argumentos. Este vector de peso se puede determinar de múltiples maneras: de forma directa de acuerdo al criterio de un experto o grupo de expertos, usando la aproximación de O'Hagan (1990), la familia de funciones de unidad básica de intervalo monotónico (BUM, siglas en inglés para Basic Unit-interval Monotonic) (Yager, 2009) y la función $f(x)=x^{r}$ que hace referencia al carácter actitudinal del tomador de decisiones (Yager, 2009).

Paso 4: Se hace el tratamiento de la información obtenida con el operador seleccionado.

Paso 5: En este último paso se ordena la información, ya sea de forma descendente o ascendente según las características del operador usado, y se ordenan las alternativas para seleccionar aquella cuyo resultado sea mayor.

El caso de ejemplo a desarrollar es un problema de selección de personal para un cargo X y que tiene un número de candidatos $C_{n}$ con un número de habilidades ya determinadas $H_{n}$. Como esas habilidades se pueden interrelacionar, se decide el uso del operador BON-OWA.

Paso 1. En un proceso de decisión se asume que ser un jefe de recursos humanos requiere completar el proceso de selección entre cinco candidatos $\mathrm{C}_{1}, \mathrm{C}_{2}, \mathrm{C}_{3}, \mathrm{C}_{4}$ y $\mathrm{C}_{5}$, y para ello necesita saber cómo se interrelacionan las siete habilidades exigidas: $\mathrm{H}_{1}$ (Programación), $\mathrm{H}_{2}$ (Matemáticas), $\mathrm{H}_{3}$ (Diseño web), $\mathrm{H}_{4}$ (Relaciones interpersonales), $\mathrm{H}_{5}$ (Análisis), $\mathrm{H}_{6}$ (Desarrollo de Software) y $\mathrm{H}_{7}$ (Redes sociales), entre cada uno de ellos.

Paso 2. A partir de esta información se construye la matriz de ponderaciones de los argumentos $a_{i}$, que se asume que es obtenida por información previamente recolectada en pasos anteriores del proceso de selección (Tabla 1).

Tabla 1. Ponderaciones de las habilidades.

\begin{tabular}{llllllll}
\hline & $\mathbf{H}_{\mathbf{1}}$ & $\mathbf{H}_{\mathbf{2}}$ & $\mathbf{H}_{\mathbf{3}}$ & $\mathbf{H}_{\mathbf{4}}$ & $\mathbf{H}_{\mathbf{5}}$ & $\mathbf{H}_{\mathbf{6}}$ & $\mathbf{H}_{\mathbf{7}}$ \\
\hline $\mathbf{C}_{\mathbf{1}}$ & 0.5 & 0.4 & 0.5 & 1.0 & 1.0 & 0.8 & 0.4 \\
$\mathbf{C}_{\mathbf{2}}$ & 0.7 & 0.5 & 0.5 & 0.5 & 0.7 & 0.6 & 0.8 \\
$\mathbf{C}_{\mathbf{3}}$ & 0.3 & 0.2 & 0.8 & 1.0 & 0.5 & 0.6 & 1.0 \\
$\mathbf{C}_{\mathbf{4}}$ & 0.3 & 0.2 & 0.8 & 0.7 & 0.7 & 0.5 & 1.0 \\
$\mathbf{C}_{\mathbf{5}}$ & 0.5 & 0.8 & 0.2 & 0.3 & 1.0 & 0.6 & 1.0 \\
\hline
\end{tabular}

Fuente: Elaboración propia. 
Paso 3. Asimismo, el jefe de personal determina la importancia de cada característica dentro del proceso de selección, que es el vector de peso $\boldsymbol{\alpha}_{\boldsymbol{i}}$. Así, los vectores de peso para cada habilidad son los siguientes: $\alpha_{1}=0.9 \alpha_{2}=0.6, \alpha_{3}=0.7 \alpha_{4}=0.5 \alpha_{5}=0.7 \alpha_{6}=0.9 \alpha_{7}=0.6$.

Paso 4. Con la información de entrada disponible se realiza su tratamiento matemático. Para mostrar cómo se desarrolla el proceso se tomará las ponderaciones de las habilidades del candidato $\mathrm{C}_{1}$ :

$$
\begin{aligned}
f_{\mathrm{C} 1}(\mathrm{H} 1, \ldots, \mathrm{H} 7) & =(0.5) \times(0.9)+(0.4) \times(0.6)+(0.5) \times(0.7)+(1.0) \times(0.5)+(1.0) \times(0.7) \\
& +(0.8) \times(0.9)+(0.4) \times(0.6)=3.2
\end{aligned}
$$

El resultado para el candidato $\mathrm{C}_{1}$ es 3.2. Este procedimiento se lleva a cabo con cada uno de los candidatos restantes, los resultados obtenidos son los siguientes:

$$
f_{\mathrm{C} 2}(\mathrm{H} 1, \ldots, \mathrm{H} 7)=3.04 \quad f_{\mathrm{C} 3}(\mathrm{H} 1, \ldots, \mathrm{H} 7)=3 \quad f_{\mathrm{C} 4}(\mathrm{H} 1, \ldots, \mathrm{H} 7)=2.9 \quad f_{\mathrm{C} 5}(\mathrm{H} 1, \ldots, \mathrm{H} 7)=
$$

Paso 5. Con los resultados definitivos se hace la ordenación de los candidatos en forma descendente, donde el primero es la opción de mayor preferencia para ocupar el cargo. La ordenación es la siguiente:

$$
\mathrm{C}_{5}>\mathrm{C}_{1}>\mathrm{C}_{2}>\mathrm{C}_{3}>\mathrm{C}_{4}
$$

Para hacer la aplicación con el operador BON-OWA se definen los parámetros $r$ y q, que son iguales a 1 para cada uno. En este caso, los primeros tres pasos son iguales para este operador. A continuación, se describe detalladamente el paso 4.

Paso 4. Se definen los vectores $\left(V^{i}\right)$ para cada uno de los candidatos. Para mostrar cómo se hace, se tomarán las ponderaciones de las habilidades del candidato $\mathrm{C}_{1}$ y se crearán los siete vectores:

$$
\begin{array}{ll}
C^{1} V^{1}=(0.4+0.5+1.0+1.0+0.8+0.4) & C^{1} V^{2}=(0.5+0.5+1.0+1.0+0.8+0.4) \\
C^{1} V^{3}=(0.5+0.4+1.0+1.0+0.8+0.4) & C^{1} V^{4}=(0.5+0.4+0.5+1.0+0.8+0.4) \\
C^{1} V^{5}=(0.5+0.4+0.5+1.0+0.8+0.4) & C^{1} V^{6}=(0.5+0.4+0.5+1.0+1.0+0.4) \\
C^{1} V^{7}=(0.5+0.4+0.5+1.0+1.0+0.8) &
\end{array}
$$

El resultado de la suma de cada uno de los vectores se multiplica por su vector de peso, $O W A_{W}\left(C^{1} V^{i}\right)=\sum C^{1} V^{i} \times \alpha_{i}$. Los resultados obtenidos son los siguientes:

$$
\begin{aligned}
O W A_{W}\left(C^{1} V^{1}\right)= & (0.4 \times 0.6)+(0.5 \times 0.7)+(1.0 \times 0.5)+(1.0 \times 0.7)+(0.8 \times 0.9)+(0.4 \\
& \times 0.6)=2.75 \\
O W A_{W}\left(C^{1} V^{2}\right)= & (0.5 \times 0.9)+(0.5 \times 0.7)+(1.0 \times 0.5)+(1.0 \times 0.7)+(0.8 \times 0.9)+(0.4 \\
& \times 0.6)=2.96 \\
O W A_{W}\left(C^{1} V^{3}\right)= & (0.5 \times 0.9)+(0.4 \times 0.6)+(1.0 \times 0.5)+(1.0 \times 0.7)+(0.8 \times 0.9)+(0.4 \\
& \times 0.6)=2.85 \\
O W A_{W}\left(C^{1} V^{4}\right) & =(0.5 \times 0.9)+(0.4 \times 0.6)+(0.5 \times 0.7)+(1.0 \times 0.7)+(0.8 \times 0.9)+(0.4 \\
& \times 0.6)=2.70
\end{aligned}
$$




$$
\begin{aligned}
O W A_{W}\left(C^{1} V^{5}\right) & =(0.5 \times 0.9)+(0.4 \times 0.6)+(0.5 \times 0.7)+(1.0 \times 0.5)+(0.8 \times 0.9)+(0.4 \\
& \times 0.6)=2.50 \\
O W A_{W}\left(C^{1} V^{6}\right) & =(0.5 \times 0.9)+(0.4 \times 0.6)+(0.5 \times 0.7)+(1.0 \times 0.5)+(1.0 \times 0.7)+(0.4 \\
& \times 0.6)=2.48 \\
O W A_{W}\left(C^{1} V^{7}\right)= & (0.5 \times 0.9)+(0.4 \times 0.6)+(0.5 \times 0.7)+(1.0 \times 0.5)+(1.0 \times 0.7)+(0.8 \\
\times & 0.9)=2.96
\end{aligned}
$$

El resultado obtenido es multiplicado por cada una de las ponderaciones de las habilidades y sumado, su total será divido por número total de los argumentos tenidos en cuenta, BON$\operatorname{OWA}\left(C^{1} H^{1}, \ldots, C^{1} H^{n}\right)=\left(\frac{1}{n} \sum_{i} C^{1} H^{i} O W A_{W}\left(C^{1} V^{i}\right)\right)^{\frac{1}{\mathrm{r}+\mathrm{q}}}$. Los resultados obtenidos son:

$$
\begin{aligned}
& B O N-O W A\left(C^{1} H^{1}, \ldots, C^{1} H^{7}\right) \\
& =\left(\frac{((0.5 \times 2.75)+(0.4 \times 2.96)+(0.5 \times 2.85)+(1.0 \times 2,7)+(1.0 \times 2.5)+(0.8 \times 2.48)+(0.4 \times 2.96))}{7}\right)^{\frac{1}{1+1}} \\
& B O N-O W A\left(C^{1} H^{1}, \ldots, C^{1} H^{7}\right)=\left(\frac{12.352}{7}\right)^{\frac{1}{2}} \\
& B O N-O W A\left(C^{1} H^{1}, \ldots, C^{1} H^{7}\right)=(1.7645)^{\frac{1}{2}} \\
& B O N-O W A\left(C^{1} H^{1}, \ldots, C^{1} H^{7}\right)=1.3284
\end{aligned}
$$

El resultado para el candidato $C_{1}$ es $\mathbf{1 . 3 2 8 4}$. Este procedimiento se lleva a cabo con cada uno de los candidatos restantes, los resultados obtenidos son los siguientes:

$$
\begin{aligned}
& B O N-O W A\left(C^{2} H^{1}, \ldots, C^{2} H^{7}\right)=1.2610 \\
& B O N-O W A\left(C^{3} H^{1}, \ldots, C^{3} H^{7}\right)=1.2412 \\
& B O N-O W A\left(C^{4} H^{1}, \ldots, C^{4} H^{7}\right)=1.1930 \\
& B O N-O W A\left(C^{5} H^{1}, \ldots, C^{5} H^{7}\right)=1.2625
\end{aligned}
$$

Paso 5. Con los resultados definitivos se hace la ordenación de los candidatos en forma descendente, donde el primero es la opción de mayor preferencia para ocupar el cargo. La ordenación es la siguiente:

$$
\mathrm{C}_{1}>\mathrm{C}_{5}>\mathrm{C}_{2}>\mathrm{C}_{3}>\mathrm{C}_{4}
$$

A continuación, en la Tabla 2 se muestra la comparación de los resultados y las ordenaciones obtenidos por ambos operadores. Se evidencia que al usar el operador OWA la preferencia es el candidato $\mathrm{C}_{5}$ y para el operador BON-OWA es $\mathrm{C}_{1}$. Estas diferencias se deben a las características de cada uno de los operadores. Por un lado, el operador OWA nos permite solamente agregar la información y asociarla con un vector de peso para obtener una ordenación de la opción más preferida a la menos preferida. Por otro lado, el operador BON-OWA no solamente nos permite agregar la 
información y obtener su orden, sino que también establece la interrelación existente entre cada uno de los argumentos y hacer una comparación continua de los mismos. Por lo tanto, para nuestro ejemplo de selección de personal el operador BON-OWA nos permite obtener un resultado más robusto al establecer la interrelación y comparación continua de las habilidades de cada uno de los candidatos.

Tabla 2. Comparación de operadores.

\begin{tabular}{lcccc}
\hline Candidato & OWA & Ordenación & BON-OWA & Ordenación \\
\hline $\mathbf{C}_{1}$ & 3.20 & 2 & $\underline{\mathbf{1 . 3 2 8 3}}$ & $\underline{\mathbf{1}}$ \\
$\mathbf{C}_{\mathbf{2}}$ & 3.04 & 3 & 1.2609 & 4 \\
$\mathbf{C}_{\mathbf{3}}$ & 3.00 & 4 & 1.2412 & 4 \\
$\mathbf{C}_{\mathbf{4}}$ & 2.90 & 5 & 1.1929 & 5 \\
$\mathbf{C}_{5}$ & $\underline{\mathbf{3 . 2 2}}$ & $\underline{\mathbf{1}}$ & 1.2601 & 2 \\
\hline
\end{tabular}

Fuente: Elaboración propia.

Hasta este punto, se ha estudiado la teoría de toma de decisiones en incertidumbre y nos hemos enfocado particularmente en los operadores OWA y BON-OWA como métodos en la toma de decisiones en entornos inciertos. De estos operadores se han expuesto sus principales definiciones y características destacando su capacidad de agregación de información y de interrelación y comparación continua.

\section{Caso de estudio.}

Este trabajo pretende mostrar la relación entre las variables y examinar las asociaciones existentes entre ellas desde un método deductivo con el fin de obtener explicaciones particulares a partir de conclusiones generales. Bajo este método se busca explicar la relación entre las diferentes variables conexas con el propósito de analizar cómo los objetivos son priorizados en la toma de decisiones para la selección de productos a lanzarse al mercado. El caso es el siguiente:

La empresa mexicana XYZ (seudónimo utilizado porque la empresa solicitó guardar el anonimato), dedicada a la fabricación de fertilizantes agrícolas con inicios de actividad en 1984 y presencia a nivel nacional, desea realizar una inversión para poder lanzar al mercado un fertilizante cuya característica principal es ser amigable con el medio ambiente y disminuir los residuos que deja en la tierra, mantos acuíferos y canales de riego. Ante esta problemática, la alta dirección ha realizado un análisis de todas las alternativas que tienen respecto a esta temática y al final, no pueden decidirse entre 4, cuyas características cumplen con las necesidades básicas para salir al mercado. Sin embargo, no se cuenta con el capital, ni con la infraestructura física para poder comenzar la producción de los 4 productos al mismo tiempo, por lo que se debe tomar la decisión de qué producto comenzar a elaborar. Los pasos para determinar los resultados y el uso de los operadores BON-OWA serán los mismos que se detallaron en la sección 2.5 del artículo.

Paso 1. Para realizar esto, se seleccionaron 6 elementos a evaluar de cada producto, con el fin de que sean la base para la toma de decisiones. Estos elementos son.

$$
\begin{aligned}
& e_{1}=\text { tamaño del mercado objetivo } \\
& e_{2}=\text { margen de utilidad del producto } \\
& e_{3}=\text { inversion de capital inicial }
\end{aligned}
$$




$$
\begin{aligned}
& e_{4}=\text { nivel de complejidad de desarrollo } \\
& e_{5}=\text { número de personas necesarias para la nueva producción } \\
& e_{6}=\text { tiempo de vida estimado en el mercado } \\
& \text { El análisis de cada elemento es el siguiente: }
\end{aligned}
$$

a. $e_{1}$ se considera como respuesta 1 cuando el mercado es regional o 2 cuando es nacional.

b. $\quad e_{2}$ la respuesta será 1 si aporta menos del $5 \%$ a la utilidad total de la compañía, 2 entre $5 \%$ y $10 \%$ y 3 en casos mayores al $10 \%$.

c. $e_{3}$ será 1 si la inversión inicial es mayor a 75 mil dólares, 2 entre 50 mil y 75 mil dólares y 3 mayores a 50 mil dólares.

d. $e_{4}$ cuando el nivel de complejidad de desarrollo sea alto será 1 , cuando sea medio es 2 y cuando sea bajo es 3 .

e. $e_{5}$ en caso de que el número de empleados para la nueva producción sea mayor a 20 es 1,2 será entre $10-20$ y menor a 10 es 3 .

f. $e_{6}$ la respuesta es 1 cuando el tiempo de vida es menor a 5 años, 2 cuando es entre 5 y 10 años y 3 en el caso de más de 10 años.

Paso 2. Teniendo en cuenta la información proporcionada por la alta dirección se puede observar que aquel producto cuya calificación sea la mayor será el que sea lanzado al mercado por encima de los otros. Posteriormente, el departamento de producción procedió a evaluar cada uno de los productos viables y los resultados se presentan en la Tabla 3.

Tabla 3. Evaluación de los productos.

\begin{tabular}{ccccc}
\hline & $\mathbf{P}_{\mathbf{1}}$ & $\mathbf{P}_{\mathbf{2}}$ & $\mathbf{P}_{\mathbf{3}}$ & $\mathbf{P}_{\mathbf{4}}$ \\
\hline $\mathbf{e}_{\mathbf{1}}$ & 1 & 1 & 1 & 2 \\
$\mathbf{e}_{\mathbf{2}}$ & 2 & 3 & 3 & 2 \\
$\mathbf{e}_{3}$ & 3 & 3 & 2 & 2 \\
$\mathbf{e}_{\mathbf{4}}$ & 3 & 2 & 3 & 2 \\
$\mathbf{e}_{5}$ & 2 & 2 & 2 & 3 \\
$\mathbf{e}_{6}$ & 3 & 3 & 2 & 2 \\
\hline
\end{tabular}

Fuente: Elaboración propia.

Con la información presentada en la Tabla 3 se procedió a analizar de forma inicial mediante un promedio simple cada producto, obteniendo los siguientes resultados $P_{1}=2.33, P_{2}=2.50, P_{3}=$ 2.16, $P_{4}=2.16$. Esto implica que el orden es $P_{2}>P_{1}>P_{3} o P_{4}$. Siendo entonces $P_{2}$ el producto que debería salir al mercado. Sin embargo, la alta dirección decidió que no todos los elementos a evaluar tienen la misma importancia dentro de la evaluación, por lo que se decidió dar peso a cada elemento y posteriormente analizarse a través de los operadores de agregación de información.

Paso 3. Se determinan los vectores de peso $\mathrm{W}_{i}$ para cada uno de los argumentos.

La alta dirección ha determinado que los pesos para cada uno de los elementos a evaluar son los siguientes (Tabla 4). 
Tabla 4. Peso de los elementos.

\begin{tabular}{cc}
\hline Elementos & Peso \\
\hline $\mathbf{e}_{1}$ & 0.10 \\
$\mathbf{e}_{2}$ & 0.25 \\
$\mathbf{e}_{3}$ & 0.25 \\
$\mathbf{e}_{4}$ & 0.10 \\
$\mathbf{e}_{5}$ & 0.20 \\
$\mathbf{e}_{6}$ & 0.10 \\
\hline
\end{tabular}

Fuente: Elaboración propia.

Paso 4. Con las ponderaciones y los vectores de peso definidos se procede al tratamiento de la información usando la media ponderada, el operador OWA y el operador BON-OWA. Los resultados se presentan en la Tabla 5.

Tabla 5. Resultados utilizando distintos operadores de agregación de información.

\begin{tabular}{lccc}
\hline & Media ponderada & OWA & BON-OWA \\
\hline $\mathbf{P}_{\mathbf{1}}$ & 2.30 & 2.60 & 1.40 \\
$\mathbf{P}_{\mathbf{2}}$ & 2.30 & 2.70 & 1.39 \\
$\mathbf{P}_{\mathbf{3}}$ & 2.35 & 2.40 & 1.43 \\
$\mathbf{P}_{\mathbf{4}}$ & 2.25 & 2.25 & 1.39 \\
\hline
\end{tabular}

Fuente: Elaboración propia.

Paso 5. Con los resultados obtenidos se procede a hacer la ordenación de los objetivos y observar su preferencia.

Con los resultados obtenidos contamos con 4 diferentes operadores para analizar los resultados cuyo ordenamiento se presenta en la Tabla 6.

Tabla 6. Ordenamiento utilizando distintos operadores de agregación de información.

\begin{tabular}{cc}
\hline Operador & Ordenación \\
\hline Media & $P_{2}>P_{1}>P_{3}>P_{4}$ \\
Media ponderada & $P_{3}>P_{1}>P_{2}>P_{4}$ \\
OWA & $P_{2}>P_{1}>P_{3}>P_{4}$ \\
BON-OWA & $P_{3}>P_{1}>P_{2} o>P_{4}$ \\
\hline
\end{tabular}

Fuente: Elaboración propia.

\section{Resultados y discusión.}

A través de lo observado en la Tabla 6 se analiza que existen dos operadores (Media y OWA) que seleccionan a $P_{2}$ como la opción más viable a trabajar y dos operadores (Media ponderada y BONOWA) indican que debería ser $P_{3}$. De tal forma y considerando que el operador BON-OWA es el que incluye mayor información dentro de los resultados se puede afirmar que la opción $P_{3}$ es aquel producto 
que debería salir al mercado. Un dato importante también se pudiera obtener si sumamos la calificación de las ordenaciones para cada producto, en donde se daría la calificación de 4 cuando sea primer lugar a 1 cuando sea cuarto lugar; mediante esta técnica obtenemos que $P_{1}=12, P_{2}=13, P_{3}=12, P_{4}=7$.

Con estos resultados podemos observar primero que de forma general el mejor producto para lanzar al mercado sería $P_{2}$; además un dato interesante es que aun cuando nunca fue seleccionado como la mejor opción $P_{1}$ se identifica como un producto de igual importancia que $P_{3}$ en relación a su calificación general. Finalmente, consideramos que el uso de este tipo de operadores genera una visión global de las posibilidades y dependiendo de lo que se desee analizar podríamos seleccionar una opción u otra, demostrando de forma clara cómo las decisiones empresariales cuentan con un grado elevado de incertidumbre. De tal forma que, cambiando un factor de mercado o elemento dentro de la toma de decisiones éstas pueden alterarse, por lo que visualizar todos los escenarios permite mejorar las posibilidades de éxito de la decisión seleccionada y así permitir a la organización una mejor rentabilidad y permanencia dentro del mercado.

\section{Conclusiones.}

En el presente trabajo se ha estudiado la toma de decisiones en entornos inciertos. La teoría de toma de decisiones en incertidumbre se fundamenta en la aplicación de las matemáticas difusas en la toma de decisiones. En ese sentido, se hace una revisión de la evolución de la toma de decisiones difusas desde el racionamiento aproximado hasta los operadores de agregación. Dentro de los diferentes métodos propuestos para la toma de decisiones se ha profundizado en el operador OWA y en una de sus extensiones llamado el operador Bonferroni OWA. Este operador se caracteriza por hacer una agregación continua e interrelacionada de la información y obtener una ordenación de ésta al final del procedimiento. Para entender este proceso se presentan dos ejemplos numéricos que indican el paso a paso de los métodos.

Para observar la aplicación de este método se lleva a cabo un estudio de caso para la toma de decisiones estratégicas en la selección de productos para lanzar al mercado de una empresa mexicana. Mediante el uso de diferentes operadores de agregación de información se encontró que dependiendo de cuál se utilice, las opciones más viables se encontraban entre $P_{3}$ y $P_{2}$. Sin embargo, considerando que el operador que incluye mayor información a la hora de generar los resultados es el BON-OWA, se recomienda a la organización lanzar al mercado $P_{3}$. A través de este análisis, se puede observar cómo las decisiones dentro del mundo empresarial cuentan con incertidumbre, por lo que el uso de diferentes herramientas que sean adaptables y permitan visualizar mejor los escenarios existentes con el uso de operadores OWA y sus extensiones es recomendable.

En conclusión, se estudió de manera holística una metodología novedosa sobre toma de decisiones estratégicas en incertidumbre, que nos permitió integrar conceptos de la teoría administrativa y la teoría de la agregación en un caso aplicado en el que se destaca más la importancia de la información y no su medición.

Finalmente, para futuras líneas de investigación se realizará el análisis de diferentes decisiones empresariales a través de operadores de agregación de información como es el caso de decisiones financieras, legales, en ingeniería, económicas y demás, relacionadas con las diferentes áreas empresariales (Laengle et al., 2017). Adicionalmente, se planteará la extensión de los operadores de OWA mediante el uso de medias móviles (León et al., 2016; 2018a), operadores priorizados (AvilésOchoa et al, 2018), logaritmos (Alfaro-Garcia et al., 2018), entre otros. 


\section{Referencias}

Alfaro-García, V.G., Merigó, J.M., Gil-Lafuente, A.M., \& Kacprzyk, J. (2018). Logarithmic aggregation operators and distance measures. International Journal of Intelligent Systems, 33(7), 1488-1506.

Avilés-Ochoa, E., León-Castro, E., Perez-Arellano, L.A., \& Merigó, J.M. (2018). Government transparency measurement through prioritized distance operators. Journal of Intelligent \& Fuzzy Systems, 34(4), 2783-2794.

Bellman, R.E., \& Zadeh, L.A. (1970). Decision-making in a fuzzy environment. Management Science, 17(4), p.B-141-B-164.

Blanco-Mesa, F., Gil-Lafuente, A.M., \& Merigó, J.M. (2015). New aggregation methods for decisionmaking in the selection of business opportunities. In J. Gil-Aluja et al., eds. Scientific Methods for the Treatment of Uncertainty in Social Science. Girona: Springer International Publishing, pp. 3-18.

Blanco-Mesa, F., Merigó, J.M., \& Kacprzyk, J. (2016). Bonferroni means with distance measures and the adequacy coefficient in entrepreneurial group theory. Knowledge-Based Systems, 111(1), 217227.

Blanco-Mesa, F., Merigó, J.M., \& Gil-Lafuente, A.M. (2017). Fuzzy decision making: a bibliometricbased review. Journal of Intelligent \& Fuzzy Systems, 32(3), 2033-2050.

Blanco-Mesa, F., Gil-Lafuente, A.M., \& Merigó, J.M. (2018a). New aggregation operators for decisionmaking under uncertainty: an applications in selection of entrepreneurial opportunities. Technological and Economic Development of Economy, 24(2), 335-357.

Blanco-Mesa, F., Gil-Lafuente, A.M., \& Merigo, J.M. (2018b). Dynamics of stakeholder relations with multi-person aggregation. Kybernetes, 47(9), 1801-1820.

Blanco-Mesa, Fabio, Gil-Lafuente, A.M., \& Merigó, J.M. (2018c). Subjective stakeholder dynamics relationships treatment: a methodological approach using fuzzy decision-making. Computational and Mathematical Organization Theory, 24(4), 441-472.

Blanco-Mesa, Fabio, Rivera-Rubiano, J., Patiño-Hernandez, X., \& Martinez-Montaña, M. (2019). The importance of enterprise risk management in large companies in Colombia. Technological and Economic Development of Economy, 1-34. DOI: 10.3846/tede.2019.9380.

Bonferroni, C. (1950). Sulle medie multiple di potenze. Bollettino dell'Unione Matematica Italiana, $5(3-4), 267-270$.

Boran, F. E., Genç, S., Kurt, M., \& Akay, D. (2009). A multi-criteria intuitionistic fuzzy group decision making for supplier selection with TOPSIS method. Expert Systems with Applications, 36(8), 11363 11368 .

Brijs, K., Vanhoof, K., Brijs, T., \& Karlis, D. (2006). Using fuzzy set theory to assess country-of-origin effects on the formation of product attitude. In V. Torra et al. (eds.). Modeling Decisions for Artificial Intelligence. Tarragona: Springer Berlin Heidelberg, pp. 138-149.

Canós, L., \& Liern, V. (2008). Soft computing-based aggregation methods for human resource management. European Journal of Operational Research, 189(3), 669-681. 
Canós, L., Casasús, T., Liern, V., \& Pérez, J.C. (2014). Soft computing methods for personnel selection based on the valuation of competences. International Journal of Intelligent Systems, 29(12), 10791099.

Casanovas, M., Merigó, J.M. \& Torres-Martínez, A. (2014). Inteligencia computacional en la gestión del riesgo asegurador: Operadores de agregación OWA en procesos de tarificación, Madrid: Fundación MAPFRE.

Chen, C.T. (2000). Extensions of the TOPSIS for group decision-making under fuzzy environment. Fuzzy sets and systems, 114(1), 1-9.

Chen, S. M., \& Tan, J. M. (1994). Handling multicriteria fuzzy decision-making problems based on vague set theory. Fuzzy sets and systems, 67(2), 163-172.

Chen, S.J. \& Hwang, C.L. (1992). Fuzzy multiple attribute decision making: Methods and applications, Berlin, Heidelberg: Springer Berlin Heidelberg.

Doskéocil, R. (2015). Fuzzy logic: An instrument for the evaluation of project status. Revista de Métodos Cuantitativos para la Economía y la Empresa, 19, 5-23.

Garg, H. (2016). A novel correlation coefficients between Pythagorean fuzzy sets and its applications to decision-making processes. International Journal of Intelligent Systems, 31(12), 1234-1252.

Gil-Aluja, J. (1996). Towards a new paradigm of investment selection in uncertainty. Fuzzy Sets and Systems, 84(2), 187-197.

Gil-Aluja, J. (1999). Elements for a theory of decision in uncertainty (Vol. 32). Springer Science \& Business Media.

Harsanyi, J.C. (1955). Cardinal welfare, individualistic ethics, and interpersonal comparisons of utility. Journal of Political Economy, 63(4), 309-321.

Hong, D.H., \& Choi, C.H. (2000). Multicriteria fuzzy decision-making problems based on vague set theory. Fuzzy sets and systems, 114(1), 103-113.

Hwang, C.-L. \& Yoon, K. (1981). Multiple attribute decision making, Berlin, Heidelberg: Springer Berlin Heidelberg.

Klir, G.J., \& Yuan, B. (1995). Fuzzy sets and fuzzy logic: theory and applications (Vol. 574). New Jersey: Prentice Hall PTR.

Laengle, S., Loyola, G., Merigó, J.M. (2017). Mean-variance portfolio selection with the ordered weighted average, IEEE Transactions on Fuzzy Systems, 25(2), 350-362.

León-Castro, E., Avilés-Ochoa, E., \& Gil-Lafuente, A.M. (2016). Exchange rate USD/MXN forecast through econometric models, time series and HOWMA operators. Economic Computation \& Economic Cybernetics Studies \& Research, 50(4), 135-150.

León-Castro, E., Avilés-Ochoa, E., \& Merigó, J.M. (2018b). Induced heavy moving averages. International Journal of Intelligent Systems, 33(9), 1823-1839.

León-Castro, E., Avilés-Ochoa, E., Merigó, J.M., \& Gil-Lafuente, A.M. (2018a). Heavy moving averages and their application in econometric forecasting. Cybernetics and Systems, 49(1), 26-43. 
Lin, L., Yuan, X.H., \& Xia, Z.Q. (2007). Multicriteria fuzzy decision-making methods based on intuitionistic fuzzy sets. Journal of computer and System Sciences, 73(1), 84-88.

Llopis, A., \& Palacios-Marqués, D. (2017). Applications of ordered weighted averaging (OWA) operators in environmental problems. Multidisciplinary Journal for Education, Social and Technological Sciences, 4(1), 52-63.

Merigó, J.M., \& Casanovas, M. (2010). The fuzzy generalized OWA operator and its application in strategic decision making. Cybernetics and Systems, 41(5), 359-370.

Merigó, J.M., \& Gil-Lafuente, A.M. (2006). Using OWG operators in the selection of financial products. Lectures on Modelling and Simulation, 7(3), 49-55.

Merigó, J.M., \& Gil-Lafuente, A.M. (2007). Unification point in methods for the selection of financial products. Fuzzy economic review, 12(1), 35-50.

Merigó, J.M., \& Gil-Lafuente, A.M. (2008). The generalized adequacy coefficient and its application in strategic decision making. Fuzzy Economic Review, 13(2), 17-36.

Merigó, J.M., \& Gil-Lafuente, A.M. (2010). New decision-making techniques and their application in the selection of financial products. Information Sciences, 180(11), 2085-2094.

Merigó, J.M., \& Gil-Lafuente, A.M. (2011). OWA operators in human resource management. Economic computation and economic cybernetics studies and research, 45(2), 153-168.

Merigó, J.M., \& Gil-Lafuente, A.M. (2012). A method for decision making with the OWA operator. Computer Science and Information Systems, 9(1), 357-380.

Merigó, J.M. (2015). Decision-making under risk and uncertainty and its application in strategic management. Journal of Business Economics and Management, 16(1), 93-119.

O'Hagan, M. (1990). Using maximum entropy-ordered weighted averaging to construct a fuzzy neuron. In Proceedings of the 24th Annual IEEE Asilomar Conference on Signals, Systems and Computers, Pacific Grove, pp. 618-623.

Ortigosa-Hernández, M., \& Gil-Lafuente, A.M. (2016). Metodología para elaborar leyes de posibilidad de retirada del cliente: una aplicación al sector del vestido. Revista de Métodos Cuantitativos para la Economía y la Empresa, 22, 139-163.

Pérez-Arellano, L.A., León-Castro, E., Avilés-Ochoa, E., \& Merigó, J.M. (2019). Prioritized induced probabilistic operator and its application in group decision making. International Journal of Machine Learning and Cybernetics, 10 (3), 451-462.

Tzeng, G.-H. \& Huang, J.-J. (2011). Multiple attribute decision making: Methods and applications, Boca Raton: Chapman and Hall/CRC.

Vigier, H.P., Scherger, V., \& Terceño, A. (2017). An application of OWA operators in fuzzy business diagnosis. Applied Soft Computing Journal, 54, 440-448.

Wei, G.W., \& Merigó, J.M. (2012). Methods for strategic decision-making problems with immediate probabilities in intuitionistic fuzzy setting. Scientia Iranica, 19(6), 1936-1946. DOI: 10.1016/j.scient.2012.07.017. 
$\mathrm{Xu}, \mathrm{Z}$. (2008). Hybrid weighted distance measures and their application to pattern recognition. In C. Fyfe et al., eds. Intelligent Data Engineering and Automated Learning-IDEAL 2008. Daejeon: Springer Berlin Heidelberg, pp. 17-23.

$\mathrm{Xu}$, Z. (2015). Uncertain multi-attribute decision-making: Methods and applications, Berlin, Heidelberg: Springer-Verlag Berlin Heidelberg.

Yager, R.R. (1988). On ordered weighted averaging aggregation operators in multicriteria decisionmaking. IEEE Transactions on Systems, Man, and Cybernetics, 18(1), 183-190.

Yager, R.R. (1993). Families of OWA operators. Fuzzy Sets and Systems, 59(2), 125-148.

Yager, R.R. (1994). Interpreting linguistically quantified propositions. International Journal of Intelligent Systems, 9(6), 541-569.

Yager, R.R. (1996a). Quantifier guided aggregation using OWA operators. International Journal of Intelligent Systems, 11(1), 49-73.

Yager, R.R. (1996b). On the inclusion of variance in decision making under uncertainty. International Journal of Uncertainty, Fuzziness and Knowledge-Based Systems, 4(5), 401-419.

Yager, R.R. (2002). Heavy OWA operators. Fuzzy Optimization and Decision Making, 1(4), 379-397.

Yager, R.R. (2003). E-Z OWA weights. In 10th IFSA World Congress. Istambul, pp. 39-42.

Yager, R.R. (2006). Generalizing variance to allow the inclusion of decision attitude in decision making under uncertainty. International Journal of Approximate Reasoning, 42(3), 137-158.

Yager, R.R. (2009). On generalized Bonferroni mean operators for multi-criteria aggregation. International Journal of Approximate Reasoning, 50(8), 1279-1286.

Zadeh, L.A. (1965). Fuzzy sets. Information and Control, 8(3), 338-353.

Zadeh, L.A. (1975a). The concept of a linguistic variable and its application to approximate reasoning I. Information Sciences, 8, 199-249.

Zadeh, L.A. (1975b). The concept of a linguistic variable and its application to approximate reasoning II. Information Sciences, 8(4), 301-357.

Zadeh, L.A. (1975c). The concept of a linguistic variable and its application to approximate reasoning III. Information Sciences, 9(1), 43-80.

Zadeh, L.A. (1999). Fuzzy sets as a basis for a theory of possibility. Fuzzy Sets and Systems, 100(1), 934.

Zeng, S.Z., \& Su, W. (2015). Extended vikor method based on induced aggregation operators for intuitionistic fuzzy financial decision-making. Economic Computation \& Economic Cybernetics Studies \& Research, 49(4), 289-303.

Zimmermann, H.J. (1978). Results of Empirical Studies in Fuzzy Set Theory. In: Klir G.J. (eds). Applied General Systems Research. NATO Conference Series, vol 5. Springer, Boston, MA. DOI: 10.1007/978-1-4757-0555-3_21. 